\title{
Transgene Expression under the Adenoviral Major Late Promoter, Tripartite Leader Sequence and E1 Genes in Absence and Presence of Adenovirus Infection
}

\author{
Mohamed El-Mogy ${ }^{1} \&$ Yousef Haj-Ahmad ${ }^{1}$ \\ ${ }^{1}$ Department of Biological Sciences, Brock University, ON, Canada \\ Correspondence: Mohamed El-Mogy, Department of Biological Sciences, Brock University, 500 Glenridge Ave, \\ St. Catharines, ON L2S 3A1, Canada. Tel: 1-905-360-0440. E-mail: melmogy@hotmail.com
}

Received: February 6, 2012 Accepted: March 7, 2012 Online Published: November 6, 2012

doi:10.5539/jmbr.v2n1p12 URL: http://dx.doi.org/10.5539/jmbr.v2n1p12

The research is financed by the Egyptian Government and the Egyptian Cultural \& Educational Bureau in Canada

\begin{abstract}
Active gene expression driven by the adenoviral major late promoter (MLP) and the tripartite leader sequence (TPL) influenced their utilization to drive vaccines expression. It is unclear if the complete TPL sequence is required for this active expression or if it can be further boosted in presence of the adenoviral E1 genes. In this study, we investigated the effect of TPL exones and E1 genes, out of the viral genome or under adenoviral infection, on green fluorescence protein (GFP) expression driven by the MLP. Gradual increase in the transcript copies with the addition of TPL exons indicated an additive effect especially under infection. The presence of E1 genes significantly enhanced transgene transcription. Infected cells showed higher GFP transcription and translation than non-infected cells. These results indicate higher exogenous gene expression driven by the MLP can be achieved in the presence of adenoviral components other than the TPL and E1 genes.
\end{abstract}

Keywords: transgene expression, adenovirus, major late promoter, tripartite leader sequence, adenovirus E1 genes

\section{Introduction}

The adenoviral major late promoter (MLP) is known to be one of the most active viral promoters in mammalian cells (Markrides, 1999; Papadakis, Nicklin, Baker, \& White, 2004). The late phase of adenovirus infection is characterized by the production of an abundant amount of late proteins required to form and assemble the new viral capsids. Active expression in this phase is attributed to the activity of the MLP and the presence of the tripartite leader sequence (TPL). The expression of all the late viral proteins is driven by the MLP which has its full activity during the late phase of the viral infection and is transactivated by the adenoviral E1A proteins (Parks \& Shenk, 1997; Ziff \& Evans, 1978).

TPL is a 5' untranslated sequence present in all of the late, but none of the early, viral mRNA. Adenovirus serotype 5 (Ad5) leader sequence is $201 \mathrm{bp}$ formed by the splicing of three exons during the post-transcriptional modifications. TPL facilitates mRNA transport and accumulation in the cytoplasm and is responsible for the selective translation of the late viral proteins in preference to the cellular proteins (Zhang, Feigenblum, \& Schneider, 1994).

Adenovirus E1B-55K and E4-orf6 play the main role in active transport of TPL-containing mRNA from the nucleus to the cytoplasm (Bridge \& Ketner, 1990; Leppard \& Shenk, 1989) and also inhibits protein synthesis by reducing phosphorylation of the translation factor E1F2 $\alpha$ during the late phase of viral infection (Spurgeon \& Ornelles, 2009). The viral transcription sites in the nucleus contain a complex of E1B-55K and E4-orf6 (Gonzalez \& Flint, 2002). Evidence suggests that viral mRNA interacts with this complex through the ability of E1B-55K to bind RNA (Horridge \& Leppard, 1998) and facilitate its transport to the cytoplasm using the E4-orf6 proteins nuclear localization and transport signals (Dobbelstein, Roth, Kimberly, Levine, \& Shenk, 1997). Cellular mRNA transport is blocked by the same complex (Flint \& Gonzalez, 2003). Translation of any TPL-attached mRNA is eIF-4F-independent (Dolph, Racaniello, Villamarin, Palladino, \& Schneider, 1988). The 
relaxed secondary structure of TPL facilitates its function in translation initiation even when eIF-4F is inhibited (Dolph, Huang, \& Schneider, 1990).

In order to assess the effectiveness of the adenoviral TPL exones and E1 genes to achieve high transgene expression levels, driven from the MLP and independent from the adenoviral infection, we looked at the effect of these components on gene expression in non-infected as well as infected cells. This helped to understand the functional activity and the sufficiency of these components to maintain high expression levels outside of their viral genome context and infected environment.

\section{Materials and Methods}

\subsection{Plasmid Constructs}

Five plasmids were constructed based on the pUC19 backbone and all contain the MLP as a common promoter and TPL with all (pMTGA), partial (pMT1GA \& pMT1,2GA) or no exons (pMGA). One construct contains the complete TPL and the adenoviral ITRs-E1 (pE1). All of these plasmids contain the GFP expression cassette with differences in the regulatory sequences. The maps of the different GFP expression cassettes are shown in Figure 1.

\subsection{Cell Lines and Maintenance}

The used Chinese hamster ovary (CHO) cells were the subclone K1 (ATCC CCL-61) derived from the parental cell line initiated by Puck, Cieciura, and Robinson (1958). Cells were maintained as a monolayer in Petri cell culture dishes and cultured in advanced Dulbecco's Modified Eagle Medium (Advanced D-MEM: Invitrogen Corp., Gibco), containing 5\% (v/v) fetal bovine serum (FBS, PAA Laboratories Inc.), 1\% (v/v) penicillin/streptomycin (Invitrogen Corp., Gibco) and 1\% (v/v) glutamine (Invitrogen Corp., Gibco). Growing cells were incubated in a water-jacketed incubator (Fisher Scientific, Pittsburgh PA) at $37^{\circ} \mathrm{C}$ with $96 \%$ relative humidity and $5 \% \mathrm{CO}_{2}$.

\subsection{Lipofectamine 2000 Transfection}

Lipofectamine 2000 (Invitrogen) was used to transfect plasmids DNA into mammalian cells. The confluency of the monolayer was ensured to be at least $70 \%$ at the transfection time. The culture medium was replaced prior the transfection with $2 \mathrm{~mL}$ of antibiotic-free medium. The transfection mix for each well (of a 6-well plate) was prepared in $500 \mu \mathrm{L}$ by mixing plasmid DNA and Lipofectamine 2000. First, $5 \mu \mathrm{g}$ plasmid DNA was diluted in a total volume of $250 \mu \mathrm{L}$ using Opti-MEM I Reduced Serum Medium (Invitrogen Corp., Gibco). Similarly, $5 \mu \mathrm{L}$ Lipofectamine 2000 was diluted in $250 \mu \mathrm{L}$ total volume by using Opti-MEM. Both the diluted DNA and Lipofectamine 2000 were incubated at room temperature for 5 minutes then mixed together and incubated at room temperature for additional 20 minutes. The $500 \mu \mathrm{L}$ transfection mixture was added dropwise onto the well and shaken to distribute the mixture evenly. Finally, the plate was incubated at $37^{\circ} \mathrm{C}$ for 6 hours before changing the medium with the regular, antibiotic-containing, medium.

\subsection{Adenovirus and Its Infection}

Adenovirus d1309 was used in this study. The viral titre was determined by plaque assay according to the method described by Cromeans, Lu, Erdman, Humphrey, and Hill (2008). Viral infection of mammalian cells was carried out in 6-well plates by using a volume of the viral stock equivalent for the required multiplicity of infection (MOI). This viral volume was mixed with $\mathrm{PBS}++\left(0.01 \% \mathrm{CaCl}_{2} \cdot 2 \mathrm{H}_{2} \mathrm{O}\right.$ and $0.01 \% \mathrm{MgCl}_{2} \cdot 6 \mathrm{H}_{2} \mathrm{O}$ dissolved in PBS) in a total volume of $500 \mu \mathrm{L} /$ well and then added to the cell monolayer (after aspirating the medium). The 6-well plate was then incubated for 1 hour at $37^{\circ} \mathrm{C}$ with $96 \%$ relative humidity and $5 \% \mathrm{CO}_{2}$, with swirling the plate every 15 minutes. After that, $2 \mathrm{~mL}$ of the culture medium were added to each well and the plate was returned to the incubator.

\subsection{RNA/DNA Isolation}

DNA and RNA were isolated, all from the same sample, using the RNA/DNA/Protein Purification Kit (Norgen Biotek Corp.), according to the manufacturer's instructions.

\subsection{DNase Treatment of RNA}

The digestion of residual DNA in the isolated RNA samples was performed by using TURBO DNase (Ambion). Each $50 \mu \mathrm{L}$ of sample was digested in a $100 \mu \mathrm{L}$ reaction mixture containing the provided buffer and four units of TURBO DNase, with incubation at $37^{\circ} \mathrm{C}$ for 30 minutes. 


\subsection{RNA Cleaning}

All cleaned RNA samples were carried out by using the RNA CleanUp and Concentration Kit (Norgen Biotek Corp.), according to the manufacturer's instructions.

\subsection{Reverse Transcription}

Two to five hundred nanograms of total RNA was used in reverse transcription (RT) reactions. RNA was mixed with $0.5 \mu \mathrm{L}$ of $100 \mathrm{mM}$ oligo(dT) 18 primer (Sigma), and completed to a final volume of $5 \mu \mathrm{L}$ using RNase/DNase-free water (Ambion). This mixture was heated up for 5 minutes at $70^{\circ} \mathrm{C}$, then chilled to $4^{\circ} \mathrm{C}$. During the cooling step, $15 \mu \mathrm{L}$ of the RT reaction solution is added to the mixture. The added RT reaction solution contains $4 \mu \mathrm{L}$ of $5 \mathrm{X}$ First Strand Buffer (250 mM Tris- $\mathrm{HCl} \mathrm{pH} 8.3,375 \mathrm{mM} \mathrm{KCl}$ and $15 \mathrm{mM} \mathrm{MgCl}_{2}$ ), 2 $\mu \mathrm{L}$ of $0.1 \mathrm{M}$ Dithiothreitol (DTT), $1 \mu \mathrm{L}$ of $10 \mathrm{mM}$ dNTPs, $0.1 \mu \mathrm{L}$ Superscript III reverse transcriptase (Invitrogen) and $7.9 \mu \mathrm{L}$ RNase/DNase-free water (Ambion). The reaction was continued by an incubation step at $25^{\circ} \mathrm{C}$ for 5 minutes, followed with 90 minutes incubation at $42^{\circ} \mathrm{C}$ and 15 minutes incubation at $70^{\circ} \mathrm{C}$ before finally holding the reaction at $4^{\circ} \mathrm{C}$.

\subsection{Quantitative PCR}

Quantitative PCR (qPCR) was performed on a known concentration of template DNA or complementary DNA (cDNA), using the Bio-Rad iCycler thermal cycler. Specific primers within the GFP gene were used (GFP-F: 5' ATCCTGATCGAGCTGAATGG 3' and GFP-R: 5' TGCCATCCTCGATGTTGTG 3') with an amplicon size of $484 \mathrm{bp}$. The reaction mixture contained $10 \mu \mathrm{L}$ of $2 \mathrm{X}$ SYBR GREEN master mix (Bio-Rad), and $1.2 \mu \mathrm{L}$ of each primer ( $5 \mathrm{mM}$ stock). The total volume of the reaction was completed with $\mathrm{dH}_{2} \mathrm{O}$ to $20 \mu \mathrm{L}$. A 15 minutes heating at $95^{\circ} \mathrm{C}$ was used to activate the hotstart enzyme. Forty amplification cycles were performed as follow: 15 seconds at $95^{\circ} \mathrm{C}, 30$ second at $59^{\circ} \mathrm{C}$ and 1 minute at $72^{\circ} \mathrm{C}$. The reaction was kept at $57^{\circ} \mathrm{C}$ for 1 minute before starting a melting curve analysis by a $0.5^{\circ} \mathrm{C}$ increment every $10 \mathrm{sec}$ over 80 rounds. A standard curve of known plasmid concentration (10 fg to $1 \mathrm{ng}$ ) was used to determine the initial concentration of plasmid in each sample.

\subsection{GFP Fluorescence Intensity Quantification}

The fluorescence intensity of green fluorescence protein (GFP) was quantified directly from mammalian cells by measuring the relative fluorescence units (RFU) using the BioTek Synergy HT Multi-Mode Microplate Reader. Transfected cells were washed twice with PBS, lifted from the plate and counted. Fifty thousand cells per well were then transferred to a black rounded-bottom 96-well plate (Costar) in a total volume of $200 \mu \mathrm{L}$ of PBS. The RFU was then measured at an excitation wavelength of $485 \mathrm{~nm}$ and an emission wavelength of $528 \mathrm{~nm}$, using non-transfected cells as a blank.

\section{Results}

Gene expression from the different constructed plasmids was investigated in the absence and presence of adenovirus d1309. An initial experiment was carried out to optimize the viral MOI in CHO cells by using pE1 and then we used the appropriate MOI in the following gene expression evaluation from the different plasmid constructs.

\subsection{Gene Expression from $\mathrm{pE} 1$ in Cells Infected at Different MOIs}

Two sets of monolayer $\mathrm{CHO}$ cells were prepared, each set contained five groups of triplicate wells each. First, all wells were transfected with $\mathrm{pE} 1$, using Lipofectamine 2000. The transfection medium was changed 6 hours after transfection and cells were allowed to recover from the transfection for another 6 hours. Then, infection with adenovirus dl309 at MOIs of $0,1,5$ and $10 \mathrm{PFU} /$ cell was carried out. Two negative control groups were prepared; the first is $\mathrm{CHO}$ cells transfected with pUC19 and used as a control for transfected cells, while the second is pUC19 transfected and infected with the virus. Samples were collected over time $(0,12,36$ hours, 4.5, 9.5 and 14.5 days post-transfection) from the two sets where one sets was used to isolate DNA and RNA from the attached cellular monolayer, both from same sample, and the second set was used for GFP fluorescence intensity quantification. Three biological replicates were carried out and the statistical analysis of all the data obtained was performed by two way ANOVA using Tukey's test, at a significance level of $<0.05$.

Cells remained attached in monolayers with MOIs of 0 and $1 \mathrm{PFU} / \mathrm{cell}$, while cell detachment was observed with MOIs of 5 and $10 \mathrm{PFU} / \mathrm{cell}$. The amount of detached cells, on day 5 post-transfection compared to the MOI of 0 $\mathrm{PFU} /$ cell, was estimated to be $40-50 \%$ with MOI 5 PFU/cell and 80-90\% with MOI $10 \mathrm{PFU} /$ cell using a light microscope. Cell detachment seems to be proportional to the MOI. On the other hand, equal amounts of DNA were used in a qPCR reaction and the amount of DNA per CHO cell (3.1 pg/cell) (Gregory, 2012) was used to determine the plasmid copy number per cell, within the different collected samples (Figure 2). A significant (at 
$\mathrm{P}<0.05$ ) increase in plasmid copy number was observed at 12 hours post-transfection, then it decreases significantly on 36 hours post-transfection but is still significantly higher than the baseline. Insignificant increase from the baseline was reached on day 4.5 and afterwards. The same trend was observed for plasmid stability with all of the MOIs.

In addition, RT was performed on equal amounts of the DNase-treated and cleaned RNA samples. Equal volumes of the RT product were used in a qPCR reaction to quantify GFP mRNA copy numbers per cells, based on a total RNA content of $8.17 \pm 1.17$ pg per each $\mathrm{CHO}$ cell (Figure 3). Insignificant (at $\mathrm{P}<0.05$ ) increment in mRNA copies was seen when using MOI of 1 PFU/cell. MOIs of 5 and $10 \mathrm{PFU} /$ cell showed lower mRNA copies than at MOI of $1 \mathrm{PFU} /$ cell. The GFP intensity was determined in equal cell counts from each sample by measuring the RFU (Figure 4). A significant (at $\mathrm{P}<0.05$ ) increase in fluorescence intensity was obtained over 36 hours to 5.5 days post-transfection with all of the MOIs used and reached the baseline on day 9.5 and thereafter. Infection at an MOI of 1 PFU has higher expressed GFP than all the other used MOIs (0, 5 and 10 PFU/cell).

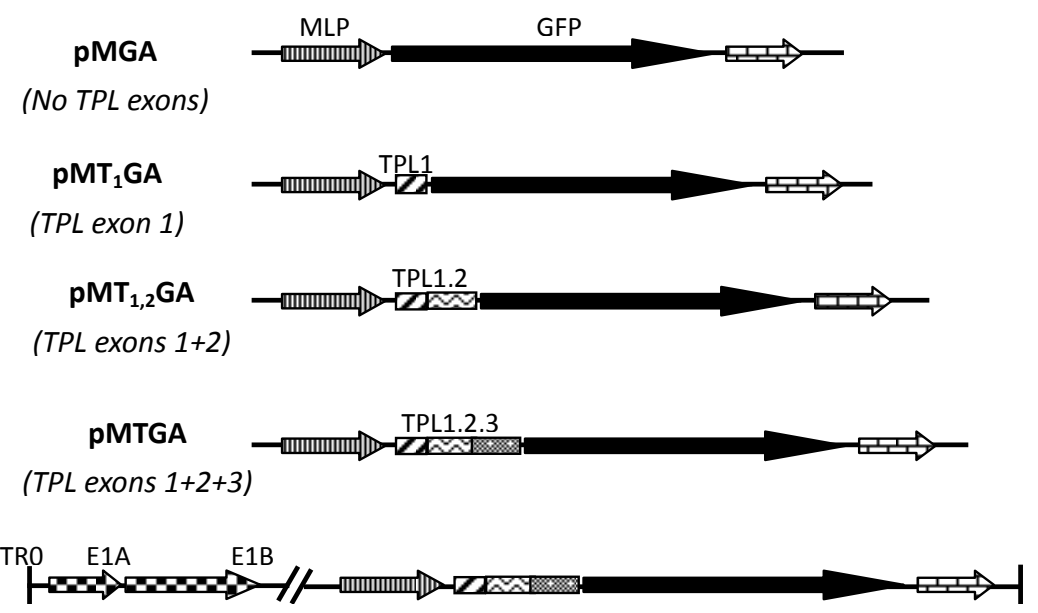

Figure 1. Schematic diagrams of the GFP cassettes and their regulatory sequences in the different plasmids. All of these sequences were constructed into the pUC19 backbone and used in all experiments in the circular plasmid form

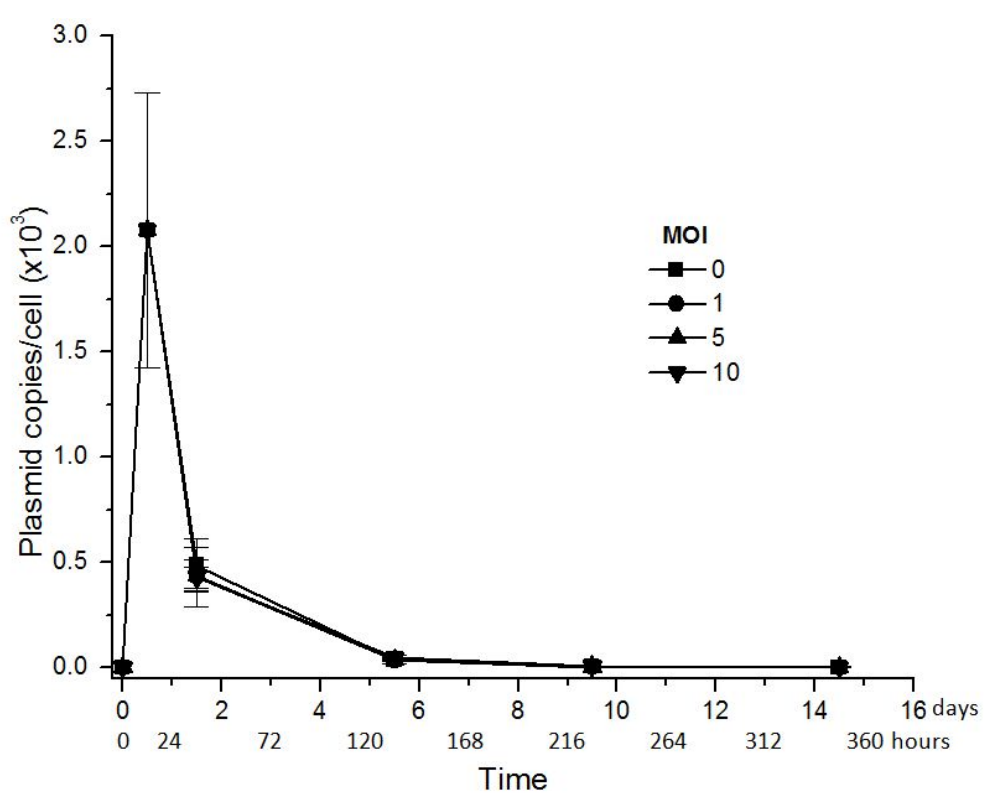

Figure 2. Copy numbers of pE1 over 14.5 days post-transfection in $\mathrm{CHO}$ cells, with infection using different MOIs. Copy numbers were obtained by qPCR using a standard curve of known plasmid DNA concentration. qPCR was performed on equal amounts of DNA isolated from collected samples. The MOIs used and their characteristic symbols are shown on the Figure 


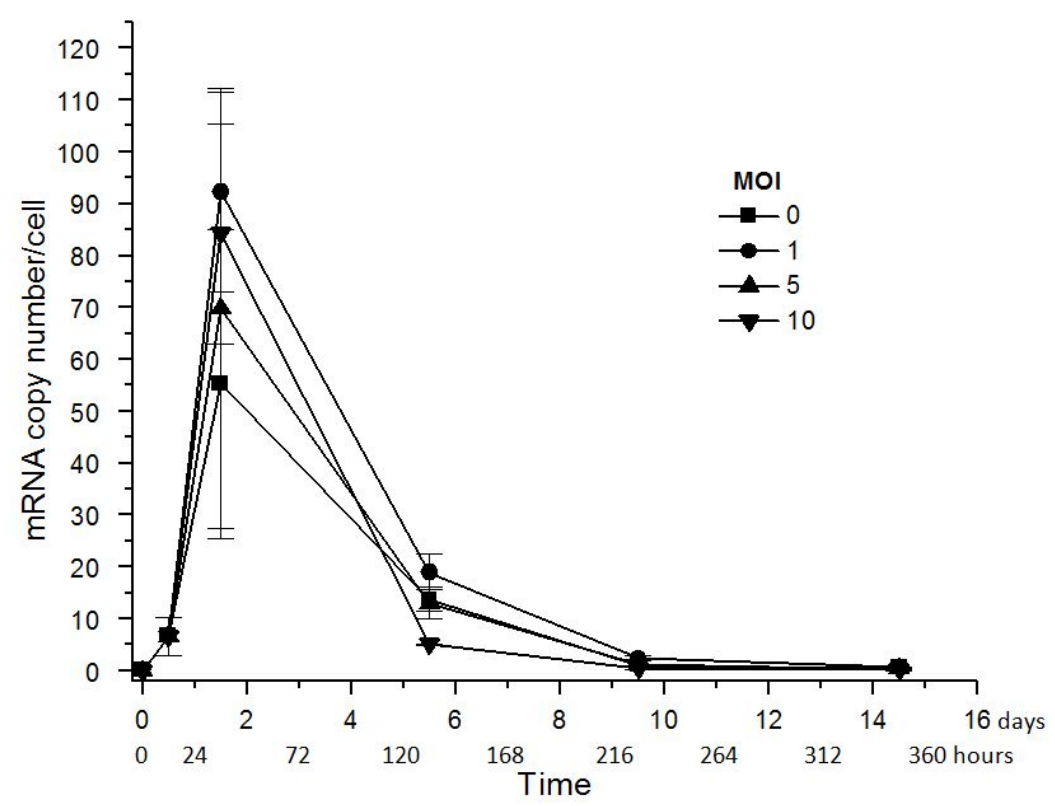

Figure 3. GFP mRNA transcripts from $\mathrm{pE} 1$ over 14.5 days post-transfection in $\mathrm{CHO}$ cells, with infection using different MOIs. Copy numbers were obtained by qPCR using a standard curve of known plasmid DNA concentration. qPCR was performed on equal volumes of RT product from equal amount of RNA isolated from collected samples. The MOIs used and their characteristic symbols are shown on the Figure

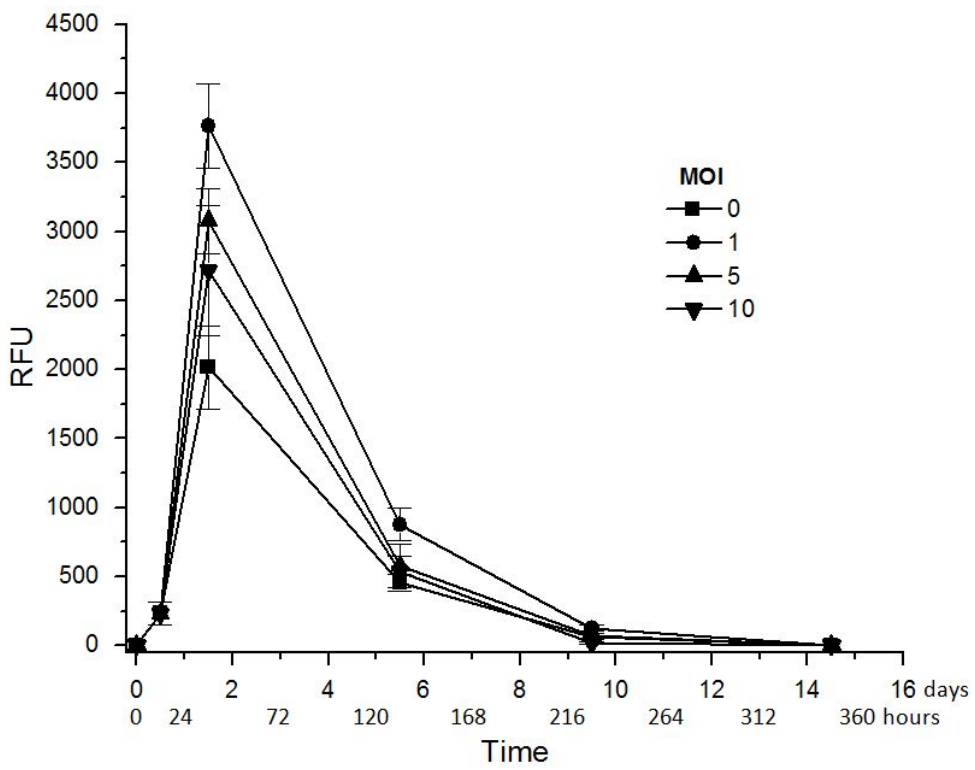

Figure 4. GFP fluorescence intensity over 14.5 days post-transfection in $\mathrm{CHO}$ cells, with infection using different MOIs. Fluorescence intensity was measured by the relative fluorescence units (RFU) of equal cell counts. The MOIs used and their characteristic symbols are shown on the Figure

\subsection{Gene Expression from the Different Constructs in Cells Infected at MOI of 1 PFU/cell}

Expression from the different plasmid constructs was investigated with infection at MOI $1 \mathrm{PFU} / \mathrm{cell}$. The lower MOI allows the side effects encountered at MOIs 5 and $10 \mathrm{PFU} /$ cell to be overcome, which is the massive cellular detachment from the monolayer. All of the plasmids were transfected into CHO cells using Lipofectamine 2000 in two triplicate groups for each plasmid, the first group to be used in RNA and DNA isolation while the second to be used in GFP fluorescence intensity quantification. The medium was changed 6 hours post-transfection to allow the cells to recover from the transfection mix. Twelve hours after transfection, 
adenovirus was infected into one group of each plasmid. In addition to the zero and 12 hours (considered as the infection's 0 time) collection times, samples were collected at 36, 84 hours, 7.5, 11.5 and 15.5 days post-transfection. Two negative controls were included; the first is CHO cells transfected with pUC19 and used as a control for transfected cells, while the second is pUC19 transfected and infected with the virus.

The isolated DNA was quantified spectrophotometrically and equal amounts were used in a qPCR reaction to determine the copy number per cell of each plasmid in the transfected and infected conditions (Figure 5). As revealed by the graphs and the statistical analysis performed on the data obtained by the two way ANOVA using Tukey's test at a significance level of less than 0.05 , all of the plasmids have an insignificant change in their stability in $\mathrm{CHO}$ cells with the transfection and the infection conditions. The maximum plasmid copy number was obtained at 12 hours post-transfection with all of the plasmids used and the two conditions. The plasmids' copy numbers remain significantly elevated on 36 hours post-transfection and reach the baseline on day 7.5 post-transfection.

The DNase treated and cleaned RNA was used in an RT reaction followed by qPCR and the copy numbers of GFP mRNA transcripts from each plasmid in the transfection and the infection conditions were calculated per cell (Figure 6). The trend for mRNA transcripts produced from the different plasmids over time in both the transfection and the infection conditions was similar, with a significant increase after 36 to 84 hours. The transcripts' levels almost reached the baseline in day 7.5 and thereafter. Despite having the same trend, the increment with the infected conditions was significantly higher than that of the transfected conditions in the five plasmids, especially over the peak period (36 to 84 hours).

The second collected group was used for GFP quantification by its fluorescence intensity and measured as the RFU in the equal cell counts from the different samples. CHO cells were used as a blank and the two negative controls (pUC19-transfected and pUC19-transfected/infected CHO cell) were included. The RFUs of GFP expressed in each plasmid with both conditions are shown in Figure 7. Three biological replicates were carried out and statistical analysis of the data obtained was performed as mentioned earlier. All of the plasmids showed significant $($ at $\mathrm{P}<0.05)$ increase in GFP fluorescence intensity after 12 hours post-transfection, in both the transfection and the infection conditions, and last until day 7.5 post-transfection. Only pMGA showed almost identical expression levels of GFP in both the transfected and the infected conditions. The rest of the plasmids showed significantly higher GFP intensity with the infection over the transfection conditions. 

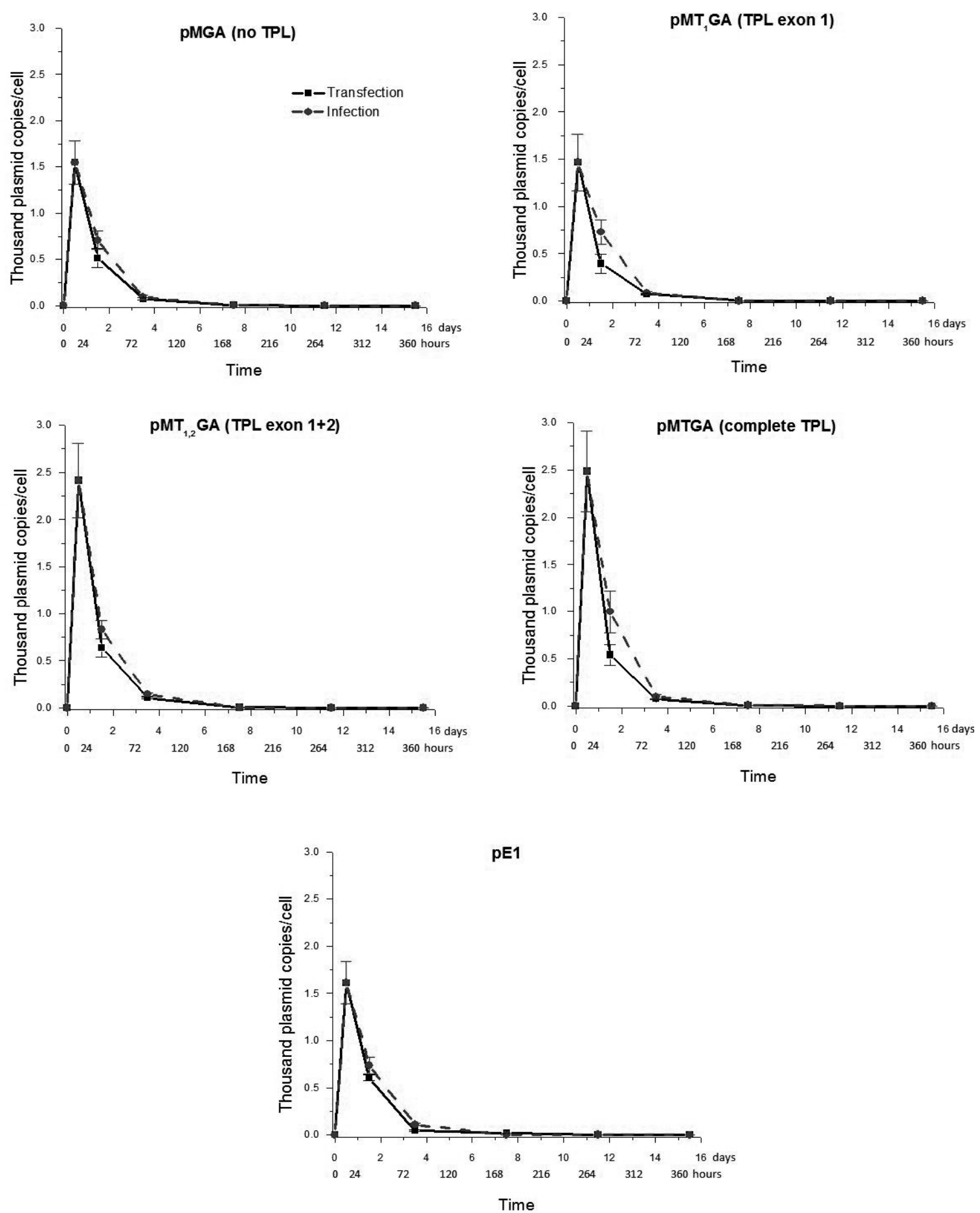

Figure 5. Copy numbers of the different plasmids over 15.5 days post-transfection in $\mathrm{CHO}$ cells, with transfection and infection (at MOI of 1 PFU/cell) conditions. Copy numbers were obtained by qPCR using a standard curve of known plasmid DNA concentration. qPCR was performed on equal amounts of DNA isolated from collected samples. Plasmids names are shown on the Figure with transfection (- - -) and infection (- - • - -) conditions 

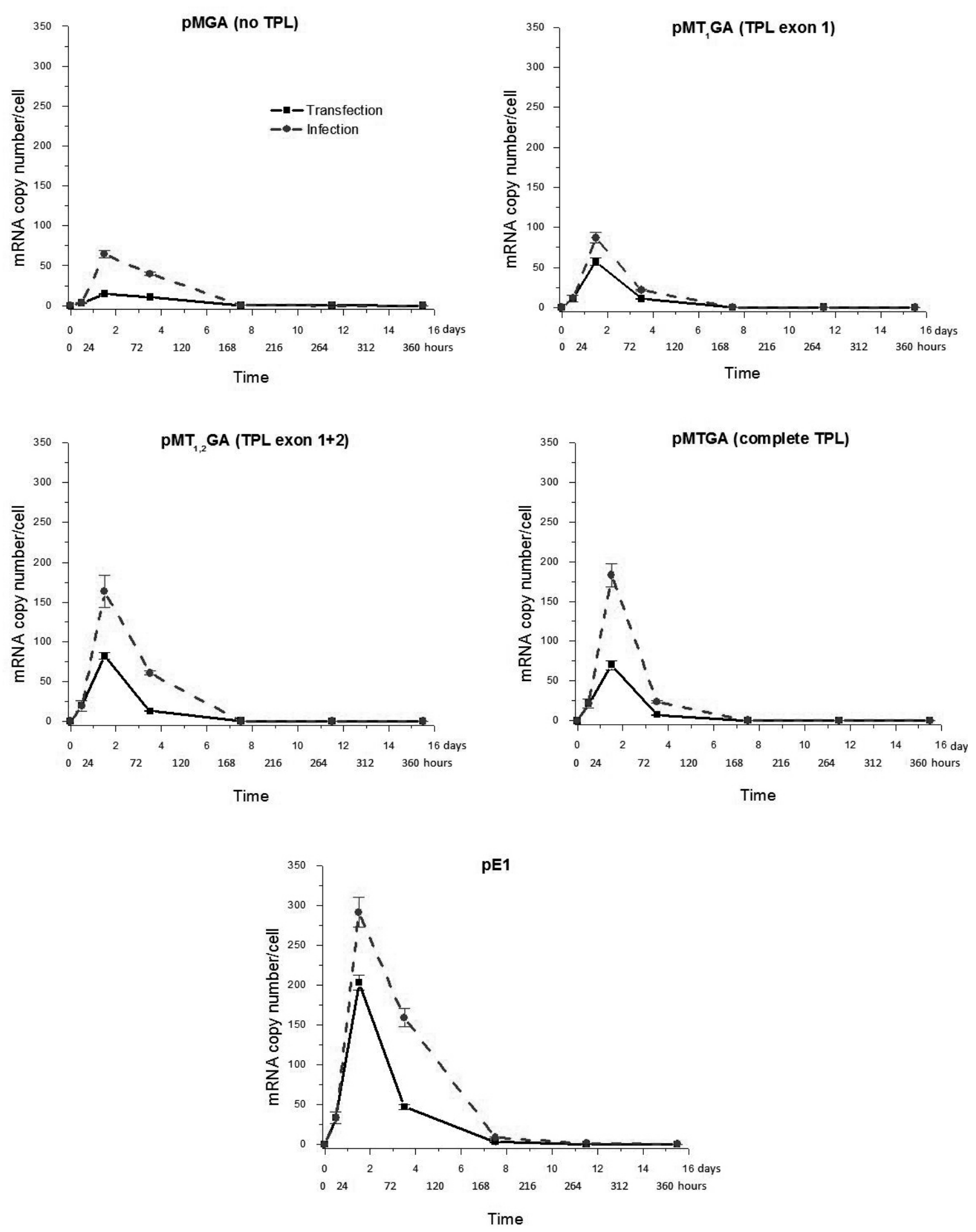

Figure 6. GFP mRNA transcripts from the different plasmids over 15.5 days post-transfection in CHO cells, with transfection and infection (at MOI of 1 PFU/cell) conditions. Copy numbers were obtained by qPCR using a standard curve of known plasmid DNA concentration. qPCR was performed on equal volumes of RT product from equal amounts of RNA isolated from collected samples. Plasmids names are shown on the Figure with transfection (-- - ) and infection (- - - -) conditions 

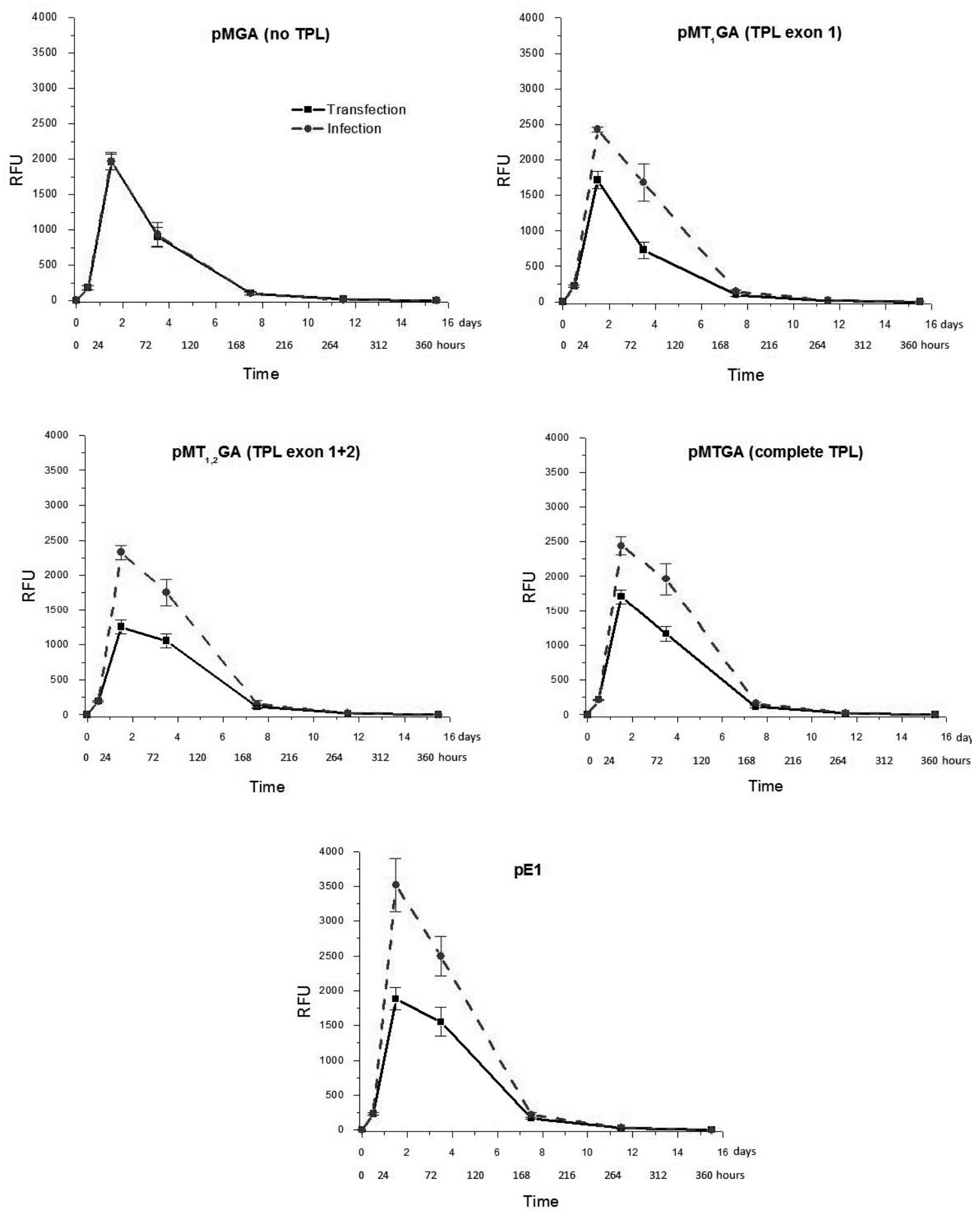

Figure 7. GFP fluorescence intensity over 15.5 days post-transfection in $\mathrm{CHO}$ cells, with transfection and infection (at MOI of $1 \mathrm{PFU} / \mathrm{cell}$ ) conditions. Fluorescence intensity was measured by the relative fluorescence units (RFU) of equal cell counts. Plasmids names are shown on the Figure with transfection (- - -) and infection $(--\bullet-)$ conditions

\section{Discussion}

Active expression driven by the adenoviral major late promoter (MLP) and tripartite leader sequence (TPL) influenced their in vivo utilization to drive the expression of vaccines (Hammond et al., 2000). The effect of TPL exons as well as E1 genes on transgene expression have not been investigated. In this study, we evaluated mRNA transcription levels and GFP translation from plasmids containing expression cassettes, with MLP and 
different TPL exons with E1 genes, in the absence and presence of adenovirus infection. We used adenovirus d1309, which has the same properties as the wild type adenovirus serotype 5 with the exception of the E3 region (Bett, Krougliak, \& Graham, 1995; Jones \& Shenk, 1978, 1979).

MOI optimization was performed for infections of CHO cells transfected with $\mathrm{pE} 1$. MOI did not have a measurable effect on transgene stability or mRNA transcription levels. At an MOI of 1 PFU/cell, GFP intensity was significantly higher than at an MOI of $10 \mathrm{PFU} /$ cell. These results concurred with our previous observations of cellular vitality and monolayer attachment. The cells remained attached at an MOI of 1 PFU/cell, similarly to the non-infected control cells, but further increases of MOI resulted in proportional detachment of cells from the monolayer.

These findings indicated that the strength of viral infection and viral protein expression directly affected transgene expression inside the cells. At higher MOIs, it appears that the competition between viral and transgene mRNA translation lowered transgene expression, while high viral loads severely affected host cells. This was in accordance with previously published data obtained from adenovirus infected HEK 293 cells. The data showed that further increases of the MOI over 5 PFU/cell did not elevate viral production (Ferreira, Alves, Gonçalves, \& Carrondo, 2005); as cellular machinery had reached its saturation point and could no longer produce more viruses. Similarly, at an MOI of $10 \mathrm{PFU} / \mathrm{cell}$ in our experiment, transgene expression was lowered by competing viral proteins, whose expression overtaxed the cellular expression machinery and affected other cellular processes. Therefore, it was concluded that use of lower MOI is more suitable for both transgene expression and cellular vitality.

The use of an MOI of $1 \mathrm{PFU} / \mathrm{cell}$ did not severely affect cellular attachment. Therefore, we were able to extend DNA, RNA and protein collection times which provided us with more detailed results. As expected from the previous optimization experiment, adenovirus infection did not affect plasmid stability over time. However, at MOI of $1 \mathrm{PFU} /$ cell, the viral yield reduced by 104 compared to an MOI of $5 \mathrm{PFU} /$ cell (Ferreira et al., 2005).

Viral infection had a positive effect on transgene transcription from the MLP, as GFP mRNA copy numbers increased after the infection. The direct effect of the viral infection on MLP activity was clearly demonstrated with the use of the constructs lacking the TPL sequence. However, the gradual increase in transgene transcript copies with the addition of TPL exons in the expression constructs indicated an additive effect. Although the inclusion of TPL exons in the expression cassette enhanced transgene mRNA expression in non-infected cells, this effect was more pronounced in adenovirus infected cells.

In the absence or presence of the adenoviral infection, about two fold increase in mRNA transcripts was observed when E1 genes were incorporated in the transgene. Most notably, the E1 proteins play a major role in the transactivation of transcription from the MLP as well as mRNA stability. Although adenovirus dl309 also encoded the E1 region, enhanced transgene expression from pMTGA did not reach same levels as pE1 after infection. This indicated that an excess amount of E1 proteins enhances transgene transcription and translation. Also, the physical presence of E1 genes in the same construct as the expression cassette can guarantee the expression of the excess E1 in the same cell at the same time. The $\mathrm{pE} 1$ plasmid increased the abundance of these proteins by increasing their gene copy number and hence, GFP expression from this construct was significantly higher than from pMTGA.

The translation of GFP mRNA also increased after viral infection, most likely as a result of the viral encoded E1B 55k and E4 orf6 proteins (Babiss, Ginsberg, \& Darnell, 1985; Blackford \& Grand, 2009; Bridge \& Ketner, 1990; Leppard \& Shenk, 1989; Pilder, Moore, Logan, \& Shenk, 1986). The only exception was GFP mRNA translation from the pMGA plasmid, which did not increase after infection. This result was expected, since the TPL sequence was absent from this transgene expression construct. On the other hand, the constructs that contained the complete TPL structure (pMTGA and pE1) showed a significant increase in GFP intensity after infection. This demonstrates the importance of the E1B 55k and E4 orf6 viral proteins. In addition, the intensity of GFP expressed from pE1 was significantly higher than that from pMTGA, mainly because of the higher transgene transcription efficiency from $\mathrm{pE} 1$.

In conclusion, these results indicate that $\mathrm{E} 1$ genes and the complete TPL have positive effect on the transcription levels driven by the MLP. The increased gene expression levels, particularly on the translational levels, in presence of adenoviral infection indicates the role of other viral proteins or elements which interact with the host cell and the transgene to up-regulate its expression. More effort is required to identify such proteins and/or elements that can restore the activity of the MLP in transgene constructs, which will in turn help to utilize these components to drive active transgene expression. 


\section{References}

Babiss, L. E., Ginsberg, H. S., \& Darnell, J. E. (1985). Adenovirus E1B proteins are required for accumulation of late viral mRNA and for effects on cellular mRNA translation and transport. Mol. Cell Biol, 5(10), 2552-2558. http://dx.doi.org/10.1128/MCB.5.10.2552

Bett, A. J., Krougliak, V., \& Graham, F. L. (1995). DNA sequence of the deletion/insertion in early region 3 of Ad5 d1309. Virus Res, 39(1), 75-82. http://dx.doi.org/10.1016/0168-1702(95)00071-W

Blackford, A. N., \& Grand, R. J. (2009). Adenovirus E1B 55-kilodalton protein: multiple roles in viral infection and cell transformation. J. Virol, 83(9), 4000-4012. http://dx.doi.org/10.1128/JVI.02417-08

Bridge, E., \& Ketner G. (1990). Interaction of adenoviral E4 and E1b products in late gene expression. Virology, 174(2), 345-353. http://dx.doi.org/10.1016/0042-6822(90)90088-9

Cromeans, T. L., Lu, X., Erdman, D. D., Humphrey, C. D., \& Hill, V. R. (2008). Development of plaque assays for adenoviruses 40 and 41. J. Virol. Methods, 151(1), 140-145. http://dx.doi.org/10.1016/j.jviromet.2008.03.007

Dobbelstein, M., Roth, J., Kimberly, W. T., Levine, A. J., \& Shenk, T. (1997). Nuclear export of the E1B $55-\mathrm{kDa}$ and E4 34-kDa adenoviral oncoproteins mediated by a rev-like signal sequence. EMBO J, 16, 4276-4284. http://dx.doi.org/10.1093/emboj/16.14.4276

Dolph, P. J., Huang, J., \& Schneider, R. J. (1990). Translation by the adenovirus tripartite leader: elements which determine independence from cap-binding protein complex. J. Virol, 64(6), 2669-2677.

Dolph, P. J., Racaniello, V., Villamarin, A., Palladino, F., \& Schneider, R. J. (1988). The adenovirus tripartite leader may eliminate the requirement for cap-binding protein complex during translation initiation. J. Virol, 62(6), 2059-2066.

Ferreira, T. B., Alves, P. M., Gonçalves, D., \& Carrondo, M. J. T. (2005). Effect of MOI and Medium Composition on Adenovirus Infection Kinetics. In F. Gòdia, \& M. Fussenegger (Eds.). Animal cell technology meets genomics (pp. 329-332). Netherlands: Springer. http://dx.doi.org/10.1007/1-4020-3103-3_65

Flint, S. J., \& Gonzalez, R. A. (2003). Regulation of mRNA production by the adenoviral E1B 55-kDa and E4 Orf6 proteins. Curr. Top. Microbiol. Immunol, 272, 287-330.

Gonzalez, R. A., \& Flint, S. J. (2002). Effects of mutations in the adenoviral E1B 55-kilodalton protein coding sequence on viral late mRNA metabolism. J. Virol, 76(9), 4507-4519. http://dx.doi.org/10.1128/ JVI.76.9.4507-4519.2002

Gregory, T. R. (2012). Animal genome size database. Retrieved January 24 2012, from http://www.genomesize.com

Hammond, J. M., McCoy, R. J., Jansen, E. S., Morrissy, C. J., Hodgson, A. L., \& Johnson, M. A. (2000). Vaccination with a single dose of a recombinant porcine adenovirus expressing the classical swine fever virus gp55 (E2) gene protects pigs against classical swine fever. Vaccine, 18(11-12), 1040-1050. http://dx.doi.org/10.1016/S0264-410X(99)00347-3

Horridge, J. J., \& Leppard, K. N. (1998). RNA-binding activity of the E1B 55-kilodalton protein from human adenovirus type 5. J. Virol, 72(11), 9374-9379.

Jones, N., \& Shenk, T. (1978). Isolation of deletion and substitution mutants of adenovirus type 5. Cell, 13(1), 181-188. http://dx.doi.org/10.1016/0092-8674(78)90148-4

Jones, N., \& Shenk, T. (1979). Isolation of adenovirus type 5 host range deletion mutants defective for transformation of rat embryo cells. Cell, 17(3), 683-689. http://dx.doi.org/10.1016/0092-8674(79)90275-7

Leppard, K. N., \& Shenk, T. (1989). The adenovirus E1B 55 kD protein influences mRNA transport via an intranuclear effect on RNA metabolism. EMBO J., 8(8), 2329-2336.

Markrides, S. C. (1999). Components of vectors for gene transfer and expression in mammalian cells. Protein Expression and Purification, 17(2), 183-202. http://dx.doi.org/10.1006/prep.1999.1137

Papadakis, E. D., Nicklin, S. A., Baker, A. H., \& White, S. J. (2004). Promoters and control elements: designing expression cassettes for gene therapy. Curr. Gene Ther, 4(1), 89-113. http://dx.doi.org/10.2174/1566523044578077 
Parks, C. L., \& Shenk, T. (1997). Activation of the adenovirus major late promoter by transcription factors MAZ and Sp1. J. Virol, 71(12), 9600-9607.

Pilder, S., Moore, M., Logan, J., \& Shenk, T. (1986). The adenovirus E1B- 55K transforming polypeptide modulates transport or cytoplasmic stabilization of viral and host cell mRNAs. Mol. Cell Biol, 6(2), 470-476. http://dx.doi.org/10.1128/MCB.6.2.470

Puck, T. T., Cieciura, S. J., \& Robinson, A. (1958). Genetics of somatic mammalian cells: iii. Long-term cultivation of euploid cells from human and animal subjects. J. Exp. Med., 108(6), 945-956. http://dx.doi.org/10.1084/jem.108.6.945

Spurgeon, M. E., \& Ornelles, D. A. (2009). The adenovirus E1B 55-kilodalton and E4 open reading frame 6 proteins limit phosphorylation of eIF2alpha during the late phase of infection. J. Virol, 83(19), 9970-9982. http://dx.doi.org/10.1128/JVI.01113-09

Zhang, Y., Feigenblum, D., \& Schneider, R. J. (1994). A late adenovirus factor induces eIF-4E dephosphorylation and inhibition of cell protein synthesis. J. Virol, 68(11), 7040-7050.

Ziff, E. B., \& Evans, R. M. (1978). Coincidence of the promoter and capped 5' terminus of RNA from the adenovirus 2 major late transcription unit. Cell, 15(4), 1463-1475. http://dx.doi.org/10.1016/0092-8674(78)90070-3 